Abstracta Iranica Abstracta Iranica

Revue bibliographique pour le domaine irano-aryen

Volume 42-43 | 2021

Comptes rendus des publications de 2019-2020

\title{
Viola Allegranzi, Valentina Laviola (eds.). Texts and Contexts. Ongoing Researches on the Eastern Iranian World (Ninth-Fifteenth $c$.)
}

\section{Sandra Aube}

\section{(2) OpenEdition \\ Journals}

Édition électronique

URL : https://journals.openedition.org/abstractairanica/53941

DOI : 10.4000/abstractairanica. 53941

ISSN : 1961-960X

Éditeur :

CNRS (UMR 7528 Mondes iraniens et indiens), Éditions de l'IFRI

Référence électronique

Sandra Aube, «Viola Allegranzi, Valentina Laviola (eds.). Texts and Contexts. Ongoing Researches on the Eastern Iranian World (Ninth-Fifteenth c.) », Abstracta Iranica [En ligne], Volume 42-43 | 2021, document 4, mis en ligne le 30 décembre 2021, consulté le 12 décembre 2022. URL : http://

journals.openedition.org/abstractairanica/53941 ; DOI : https://doi.org/10.4000/abstractairanica. 53941

Ce document a été généré automatiquement le 12 décembre 2022.

Tous droits réservés 


\title{
Viola Allegranzi, Valentina Laviola (eds.). Texts and Contexts. Ongoing Researches on the Eastern Iranian World (Ninth-Fifteenth c.)
}

\author{
Sandra Aube
}

\section{RÉFÉRENCE}

Viola Allegranzi, Valentina Laviola (eds.). Texts and Contexts. Ongoing Researches on the Eastern Iranian World (Ninth-Fifteenth c.), Rome, Istituto per l'Oriente C.A. Nallino [Pubblicazioni dell'Istituto per l'Oriente C.A. Nallino, nr. 120], 2020. 278 pp., planches couleur, index, ISBN : 978-88-97622-53-6.

1 Ce livre rassemble dix des contributions (en français ou en anglais) présentées à l'occasion d'une journée d'étude organisée le 14 septembre 2018 à Naples par Viola Allegranzi et Valentina Laviola, et portant le même titre que le présent volume.

2 Après une introduction générale ("Ongoing Researches on the Eastern Iranian World (Ninth-Fifteenth C.): A Preface to the Papers", p.11-14), l'ouvrage propose cinq premières contributions sur l'histoire culturelle du monde iranien oriental, avec un intérêt particulier porté aux IX ${ }^{\mathrm{e}}$ et XII ${ }^{\mathrm{e}}$ siècles. Les trois premiers articles sont basés sur une étude des textes et des manuscrits :

Gabrielle van den Berg interroge d'abord les modalités de transmission et d'évolution d'un texte, et décrypte les processus d'interactions entre texte, images et contexte, à travers l'exemple de l'épisode d'Alexandre le Grand (Iskandar) et la construction du mur contre les créatures de Ya'ğûğ et Ma'gûuğ ("The Wall and Beyond: Some Notes on Text, Context, and Visual Representations of Iskandar, Ya'ğūğ and Ma'ğūğ in the PreModern Persianate World", p. 15-52). 
4 Camille Rhoné-Quer relève ensuite le rôle majeur de l'Amou Darya dans l'histoire politique et culturelle de l'Orient islamique médiéval, à travers une étude du Zayn alaHbār de l'historien persan du XI ${ }^{\mathrm{e}}$ siècle Abū Sa'īd 'Abd al-Ḥayy al-Gardīzī, qu'elle compare et contextualise à la lumière de sources historiques pré-saljuqides (« Notes pour une histoire des fleuves en Orient médiéval: L'Amou Darya, une frontière du monde islamique (VII ${ }^{e}-\mathrm{XI}^{\mathrm{e}}$ siècle) ? », p. 53-77).

5 Jean-David Richaud revient ensuite sur l'historiographie du célèbre vizir Nizāàm alMulk (vers 1018-1092), puis s'intéresse aux ressorts idéologiques qui ont conduit à la transmission d'une légende nizamienne continue depuis la période médiévale, avant de finalement nuancer et relativiser la grandeur du vizir (« La légende dorée de Niẓām alMulk : Étude d'une figure de l'historiographie occidentale », p. 79-102).

6 Suivent par la suite deux articles portant sur l'histoire du décor architectural, de son contexte architectural et historique, à travers deux approches très différentes :

7 Viola Allegranzi ( Vers un réexamen des inscriptions historiques du monde iranien pré-mongol: Étude des cas des mausolées de Tim et de Termez en Ouzbékistan ", p.103-134 (cf. le compte rendu dans ce même numéro d'AbstIr)) réévalue deux inscriptions historiques aussi célèbres qu'importantes pour l'histoire culturelle et architecturale des $\mathrm{IX}^{\mathrm{e}}$-XII ${ }^{\mathrm{e}}$ siècles : l'inscription royale samanide qui encadre le portail du mausolée dit Arab-Ata à Tim (p. 105-112), et le texte de fondation du mausolée d'alḤakìm al-Tirmidì (m. 198/910 ?) à Termez (p. 112-123). En remettant en cause deux attributions pourtant communément admises et répétées depuis des décennies, Allegranzi illustre ici brillamment ce que l'approche épigraphique peut apporter à l'histoire du monde iranien oriental.

8 Andrea Luigi Corsi propose ensuite de porter un nouveau regard sur les parements en stuc provenant de l'Imāmzāda Karrār à Būzān, à l'Est d'Ispahan (“A Dating for the Archaic Stuccoes in Būzān and their Relationship with Early Abbasid Syrian-Iraqi Production", p.135-155). Son analyse attentive, nourrie et enrichie de comparaisons nombreuses avec le matériel des VIII ${ }^{\mathrm{e}}$-IXe siècles issus de différents sites syro-irakiens, conduisent Corsi à rétablir deux campagnes de décoration : une première phase dans le quatrième quart $\mathrm{du} \mathrm{VIII}^{\mathrm{e}}$ ou la première moitié $\mathrm{IX}^{\mathrm{e}}$ siècle, et une seconde en 528/1134-1135. Corsi réfute ainsi l'hypothèse d'une imitation au XII siècle de modèles antérieurs « à la manière abbasside ».

9 La seconde moitié de l'ouvrage rassemble en fait un ensemble d'études récentes sur l'histoire du site archéologique de Ghazni par l'équipe du «Islamic Ghazni Project »dont les deux éditrices du volume sont des membres actifs. C'est ainsi une formidable ressource sur l'état des connaissances actuelles sur le site :

Roberta Giunta introduit cet ensemble avec un état des lieux des travaux réalisés sur ce site archéologique depuis 1956, et fait le bilan des études en cours (« Les études sur la documentation archéologique et épigraphique de Ghazni : Résultats et nouvelles pistes de recherche ", p. 157-186).

11 Valentina Laviola livre ensuite les premiers résultats de son étude sur les décors en stuc provenant du palais ghaznévide de Ghazni ("Preliminary Analysis of Stucco Finds from the Ghaznavid Palace (Eleven-Twelfth C.) in Ghazni", p. 187-211). Sa contribution est d'un intérêt particulier puisqu'elle dévoile les premiers résultats d'une contribution originale, qui repose sur l'étude d'environ 600 fragments de décors en stuc, qu'elle 
identifie comme provenant principalement de zones du palais de Mas'ūd III (r. 1099-1115) dont aucun élément décoratif n'était jusqu'à présent connu.

Arturo Annucci présente des monnaies découvertes dans le palais ghaznévide de Ghazni ("Islamic Coins from the Ghaznavid Palace in Ghazni: A General Overview", p. 213-224). L'auteur identifie trois phases principales: une première période correspondant à la majeure phase de construction du palais (mi-XI ${ }^{e}$-début XII ${ }^{\mathrm{e}}$ siècle) ; une seconde correspondant à une phase de destruction/construction/redestruction du palais (début XII ${ }^{\mathrm{e}}$-première moitié du XIII ${ }^{\mathrm{e}}$ siècle) ; puis la période d'abandon du palais, courant de l'époque post-mongole à la période moderne.

Martina Massullo met la lumière sur une sépulture familiale: celle du prince timouride Ulug Beg b. Sulțān Abū Sa'īd (m. 907/1501-1502) et de son fils 'Abd al-Razzāq (m. 918/1512-1513), situé près du tombeau du Ghaznévide Mahmūd (r. 421/1030) sur la colline de Rawda ("Traces épigraphiques de l'élite timouride à Ghazni (Afghanistan) : Les textes commémoratifs d'Ulug Beg et 'Abd al-Razzāq ", p. 225-247). Elle propose une analyse précise et rigoureuse des inscriptions persanes inscrites sur la stèle funéraire dédiée aux deux souverains. L'auteur en donne une transcription complète ainsi qu'une traduction en français, analyse et contextualise avec soin ce matériel original qu'elle date du milieu du $\mathrm{XVI}^{\mathrm{e}}$ siècle. Cette plaque en albâtre constitue l'un des rares témoignages épigraphiques de la présence de l'élite timouride à Ghazni. L'inscription de 'Abd al-Razzāq s'avère même être le seul document de première main à mentionner les titres honorifiques du Timouride.

L'ouvrage se referme enfin sur la riche étude de Michele Bernardini sur une période méconnue de l'histoire de Ghazni («Les Qarawnas à Ghazni, XIII -XIVe siècles", p. 249-267). L'auteur plonge dans les sources historiques pour restituer la place des Qarawnas, en retraçant une l'histoire de Ghazni depuis l'invasion gengiskhanide en 618/1221, jusqu'à l'arrivée de Timour à la fin du XIV ${ }^{\mathrm{e}}$ siècle.

Chaque article est suivi de sa bibliographie, puis d'une série de planches en couleur qui présentent, la plupart du temps, un matériel méconnu et propre à constituer une ressource particulièrement intéressante. Le livre s'achève sur un index des noms, fort utile. En rassemblant un panel d'études récentes aux approches très complémentaires, ce volume, qui marque un renouveau de la recherche sur l'histoire du monde iranien oriental, avec un intérêt particulier pour les $\mathrm{IX}^{\mathrm{e}}-\mathrm{XII}{ }^{\mathrm{e}}$ siècles d'une part, et pour l'histoire de Ghazni d'autre part, est une référence notable dans ce domaine.

\section{AUTEURS}

SANDRA AUBE

CNRS, CeRMI, Paris 